\title{
Research of the probability of the "flip" of approximating function during the processing of measurement results
}

\author{
Vladimir Marchuk ${ }^{1, *}$, Dmitry Chernyshov ${ }^{1}$, Ilya Sadrtdinov ${ }^{1}$, and Alexander Minaev ${ }^{1}$ \\ ${ }^{1}$ Don State Technical University, 344000 Rostov-on-Don, Russia
}

\begin{abstract}
The paper presents the results of the studies of the probability of a "flip" of the approximating function when processing the measurement results under conditions of a priori uncertainty about the signal function and the statistical characteristics of additive noise. It is analytically proved that the confidence intervals of the probability of the absence and the presence of a "flip" are equal, which is confirmed by the experimental results. The dependences of the "flipping" of the approximating function on the sample length, the dispersion of additive noise and the rate of change of the function itself are obtained.
\end{abstract}

\section{Intoduction}

When processing the results of measurements under conditions of a priori uncertainty about the function of the useful signal and the statistical characteristics of additive noise, as follows from the results of literary sources, the splitting of the initial measurement implementation into intervals is used. Then approximation by a polynomial no higher than the second order follows[1-7] is applied. The useful signal function is not known a priori, but the requirement for Andersen smoothness is imposed on it [8]. E.g. at some interval it should be approximated by a polynomial not higher than the second order.

In [9-13], taking into account the limitations above, the method of reproduction of estimates was developed, where the approximation intervals were chosen randomly. A comparative analysis of the new method with known ones[9] showed its high efficiency, but with an increase in the dispersion of additive noise, the root-mean-square error in extracting the useful signal increases. Studies of the reasons for the increase in the error of extraction of the useful signal [9] made it possible to solve this problem. The increase in the mean-square error of the extraction of the useful signal is explained by the effect of the "flipping" of the approximating function, as shown in Figure 1.

\section{Research}

Thus, the reason for the increase in the extraction error of the useful signal was established, and recommendations were made for its reduction, which consist of the following:

\footnotetext{
*Corresponding author: marchuk@sssu.ru
} 
1. To determine the interval where the approximating function is "flipped".

2. Replace the approximation function with the mirror function relative to the original one.

In [9], it was shown that the determination and correction of the "flipping" of the approximating function can significantly improve the accuracy of extracting the useful signal. However, each of the listed recommendations is a separate independent task that requires detailed analysis and its own solution.

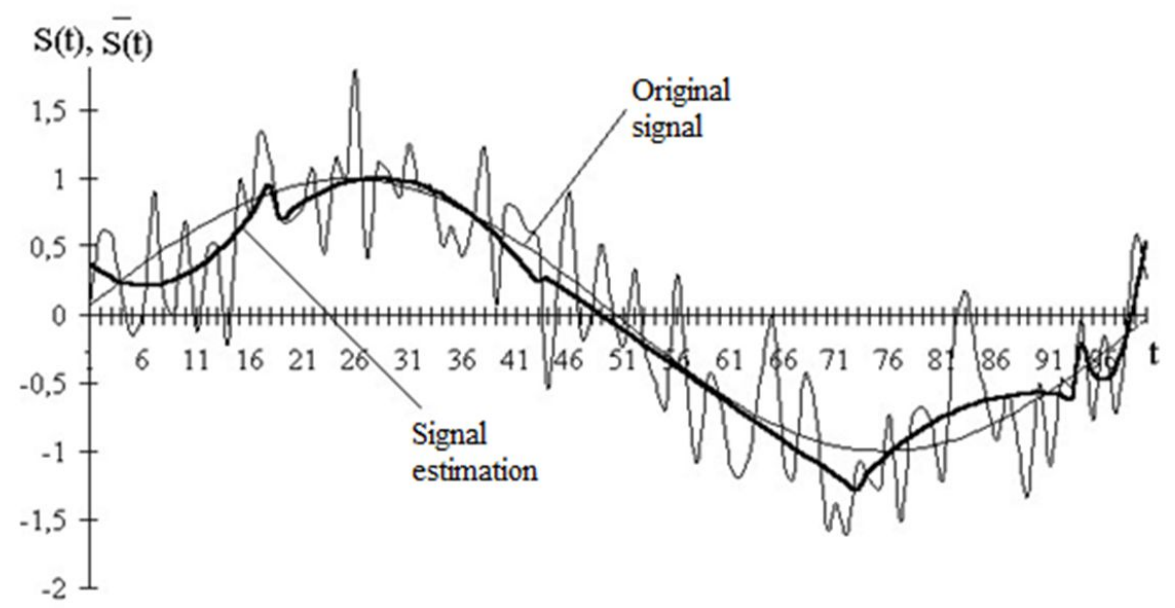

Fig.1. An example of one of the estimates in the processing of measurement results.

\section{Results}

In this paper, we present the results of the studies of the probability of the appearance of the "flipping" effect of the approximating function depending on the sample size and the dispersion of additive noise. The second-order polynomial is selected as the model of the desired signal.

$$
S(t)=a+b t+c t^{2}
$$

Gaussian noise with different dispersions was used as additive noise. The studies were carried out on samples of various lengths with 1000 realizations with the subsequent calculation of the mean value of the "flip" probability and the magnitude of the spread (the mean value variance).

First, let's consider the solution of this problem analytically. Suppose that we carried out $N$ tests, as a result of which $N_{p}$ approximations without a "flip" and $N_{n}$ with a "flip" were obtained. $N$ tests were repeated $k$ times. In this way

$$
N=N_{p}+N_{n}
$$

Then the average value of the approximations without a "flip" is

$$
\overline{m_{p}}=\frac{\sum_{i=1}^{k} N_{p i}}{k},
$$


And with a "flip"

$$
\overline{m_{n}}=\frac{\sum_{i=1}^{k} N_{n i}}{k}
$$

Similarly, we write the expressions for the dispersion of the presence of "flip" and its absence

$$
\sigma_{p}^{2}=\frac{\sum_{i=1}^{k}\left(N_{p i}-\overline{m_{p}}\right)^{2}}{k-1}, \sigma_{n}^{2}=\frac{\sum_{i=1}^{k}\left(N_{n i}-\overline{m_{n}}\right)^{2}}{k-1} .
$$

Let's consider the values of variances in the presence and absence of "flip". To do this, consider the expression

$$
\left(N_{p i}-\overline{m_{p}}\right)^{2}
$$

Considering $N=N_{p}+N_{n}$, we denote that $N_{p}=N-N_{n}$. Then

$$
\begin{aligned}
\left(N_{p i}-\overline{m_{p}}\right) & =\left(N_{i}-N_{n i}-\overline{m_{p}}\right)=\left(N_{i}-N_{n i}-\frac{\sum_{i=1}^{k} N_{p i}}{k}\right)= \\
& =\left(N_{i}-N_{n i}-\frac{\sum_{i=1}^{k} N_{i}}{k}+\frac{\sum_{i=1}^{k} N_{n i}}{k}\right)
\end{aligned}
$$

since the sum

$$
\frac{\sum_{i=1}^{k} N_{i}}{k}=N_{i}
$$

Due to the fact that the number of tests in each sample are equal and does not change, after simple transformations, we obtain that

$$
\sigma_{p}^{2}=\sigma_{n}^{2}
$$

Thus, the spread of values in the presence of "flip" and in its absence does not change, which is understandable, due to the fact that only two conclusions are possible, either there is no "flip", or there is.

We now consider the dependence $\overline{m_{p}}, \sigma_{p}^{2}$ on the sample length and the variance of the additive noise component (consideration of the dependence $\bar{m}_{n}$ and $\sigma_{n}^{2}$ separately makes no sense, since the average has an inverse relationship, and the variances are equal, which was proven above). 
Figure 2 shows the obtained dependences $\overline{m_{p}\left(\sigma^{2}\right)}$ for different values of samples $(10$, $20,50,100)$. The number of tests for each case remained constant and equal to 1000 .

The analysis of the obtained dependences allows us to conclude that with an increase in the dispersion of additive noise, the likelihood of the absence of "flip" decreases almost linearly, and with decreasing sample length, the likelihood of "flip" increases even with relatively small dispersions of additive noise. The obtained results were carried out with a useful signal in the form of a parabola with a slight change in the slope. With an increase in the slope of the useful signal model, the probability of a "flip" decreases significantly as the variance of the additive noise component changes.

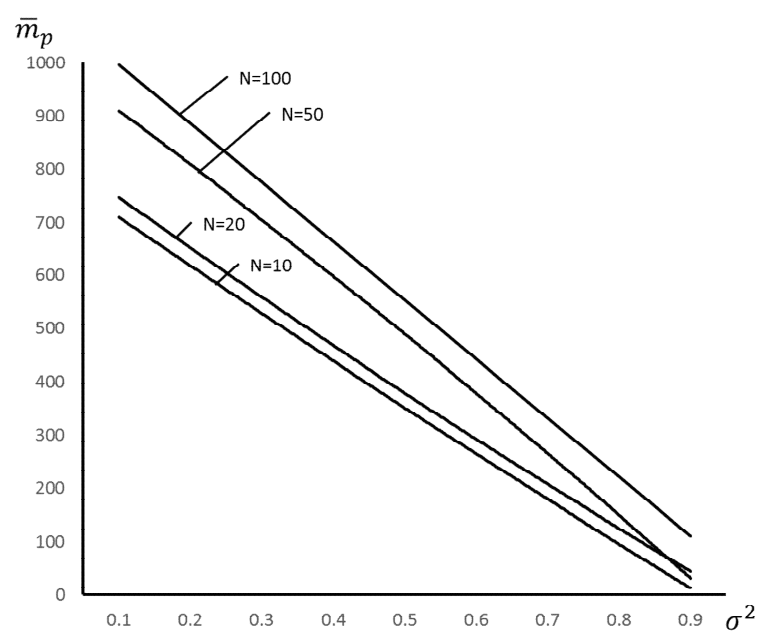

Fig. 2. Dependency of $\overline{m_{p}}$ on a sample length $N$ and variance of additive noise component $\sigma^{2}$

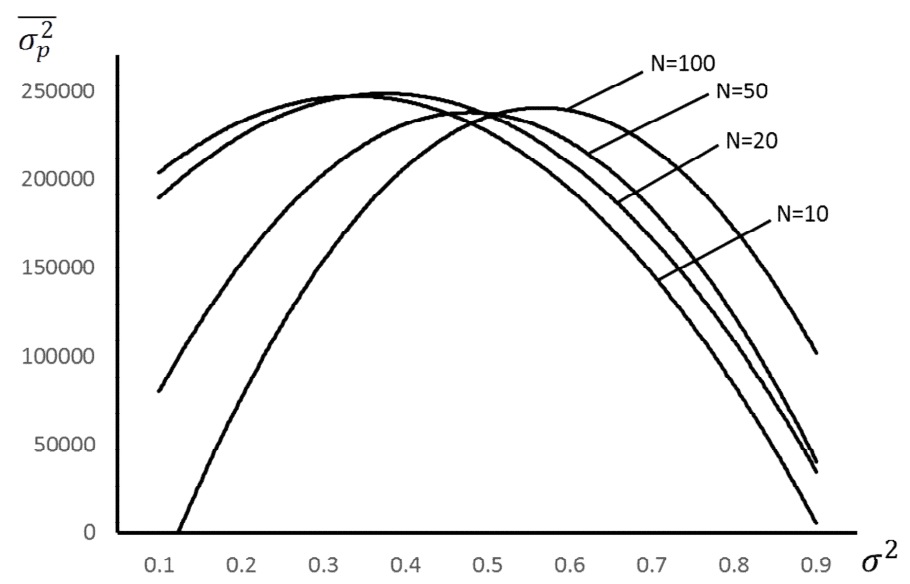

Fig. 3. Dependency of $\sigma_{p}^{2}$ on a sample length $N$ and variance of additive noise component $\sigma^{2}$

Figure 3 shows the dependences of the variance estimate of the average probability of the absence of a "flip" on the variance of the noise component and the sample length. 


\section{Conclusion}

Thus, on the basis of the conducted research the following conclusions can be made:

1. It is analytically proved that the dispersion of the values of probabilities of the presence of a "flip" and in its absence are equal.

2. With an increase in the dispersion of additive noise, the probability of the absence of a "flip" decreases almost linearly, and with a decrease in the sample length, the probability of "flip" increases even with relatively small dispersions of additive noise.

3. The dispersion of the average probability increases with increasing dispersion of the noise component, and decreases with increasing probability of "flip" with large variances of the noise component.

4. Analysis of the obtained results allows to conclude that it is necessary to eliminate the probability of a "flip", by determining and eliminating it.

\section{References}

1. J. Bendat, A. Pirsol, Applied analysis of random data (Mir, Moscow, 1989)

2. M. Kendall, J. A. Stuart, Statistical findings and communication (Science, Moscow, 1973)

3. S.M. Perevertkin, A.V. Kantor, N.F. Borodin, T.S. Shcherbakova, Onboard telemetry equipment of spacecraft (Mashinostroenie, Moscow, 1977)

4. E Hennan. Analysis of time series: Trans. from English (Science, Moscow, 1964)

5. J. Olivertti, Trend Analysis and Time Series. User's manual (1975)

6. Electronic textbook on statistics (StatSoft, Moscow, 2012) [Online] Available http://www.statsoft.ru/home/textbook/

7. M. A. Budin, IEEE Transactions on systems, MAN, and cybernetics, 4, 371 (1973)

8. T. Anderson, Statistical analysis of time series (Mir, Moscow, 1976)

9. V.I. Marchuk, Primary processing of measurement results with a limited amount of a priori information: Monograph (Publishing house TRGU, Taganrog, 2003)

10. V.I. Marchuk, Measuring equipment, 12, 3 (2003)

11. V.I. Marchuk, K.E. Rumyantsev, Aerospace Instrument Engineering, 4, 48 (2004)

12. V.I. Marchuk, G.R. Saakyan, Review of Applied and Industrial Mathematics, 9, 1, 126 (2002)

13. V.I. Marchuk, O.V. Saakyan, G.R. Saakyan, News of universities. North Caucasus region. Technical science, 3, 67 (1999) 